\title{
Microbial Strains and Bioactive Exopolysaccharide Producers from Thai Water Kefir
}

\author{
Vijitra Luang-In ${ }^{1 *}$, Worachot Saengha ${ }^{1}$, Manatchanok Yotchaisarn ${ }^{1}$, Monika Halaslova ${ }^{2}$, Piyachat Udomwong ${ }^{3}$, and \\ Sirirat Deeseenthum ${ }^{1}$ \\ ${ }^{1}$ Natural Antioxidant Innovation Research Unit, Department of Biotechnology, Faculty of Technology, Mahasarakham University, \\ Khamriang, Kantarawichai, Mahasarakham 44150, Thailand \\ ${ }^{2}$ Institute of Biotechnology and Food Science, Faculty of Chemical and Food Technology, Slovak University of Technology in Bratislava, \\ Radlinského 9, 81237 Bratislava, Slovakia \\ ${ }^{3}$ International College of Digital Innovation, Chiang Mai University, Chiang Mai 50200, Thailand
}

Received: May 1, 2018 / Revised: June 21, 2018 / Accepted: July 14, 2018

\begin{abstract}
The aims of this novel work were to determine the microbial strains and exopolysaccharide (EPS) producers in water kefir from Nakhon Ratchasima Province, Thailand. Thirty-three microbial strains were identified using 16S rRNA gene analysis consisting of 18 bacterial strains, as 9 strains of acetic acid bacteria (AAB), 9 strains of lactic acid bacteria (LAB), and 15 yeast strains. All bacteria were able to produce EPS with a diverse appearance on agar media containing different sugars at a concentration of $8 \%$. Culture supernatants from $\mathrm{AAB}$ and $\mathrm{LAB}$ showed 31-64\% 2,2-diphenyl-1-picrylhydrazyl (DPPH) scavenging activity with the highest antioxidant activity of $64 \%$ from Acetobacter pasteurianus WS3 and WS6. Crude EPS from A. pasteurianus WS3 displayed the highest ferric reducing antioxidant power at $280 \mathrm{mM} \mathrm{FeSO} / \mathrm{g} \mathrm{EPS}$, greatest anti-tyrosinase activity at $20.35 \%$, and highest EPS production of $1,505 \mathrm{mg}$ EPS/L from $8 \%$ sucrose. These microbes offer beneficial health implications and their EPSs can be used as food additives and cosmetic ingredients.
\end{abstract}

Keywords: Antioxidant, exopolysaccharide, kefir, lactic acid bacteria, tyrosinase

\section{Introduction}

Functional foods have become increasingly popular, with homemade fermented products gained attention of health conscious consumers worldwide [1]. In Thailand, the majority of the population still live in rural areas and the villagers ferment foods including Thai indigenous vegetables, fruits or fish using local wisdom passed down from generation to generation. In the past 10 years, kefirs have become more widely recognized as alternative fermented products that Thai house-holds can easily prepare at low cost from drinking water con-

\section{*Corresponding author}

Tel: $+66(0) 43754085$, Fax: $+66(0) 43754086$

E-mail: vijitra.l@msu.ac.th

๑ 2018, The Korean Society for Microbiology and Biotechnology taining brown sugar fermented with kefir grain for only 1-3 days at room temperature and this is called water kefir. In addition, milk kefir can also be prepared by inoculating cow milk with kefir grains [2]. Kefirs are light-sour, alcoholic and aromatic drinks made from kefir grains as starter cultures, consisting mainly of lactic acid bacteria (LAB), acetic acid bacteria (AAB) and yeasts [3, 4]. Evidence of the beneficial health properties of kefirs in addition to their nutritive values (rich in amino acids, vitamin $\mathrm{K}, \mathrm{B} 12$, organic acids) including antioxidation, anti-inflammation, and antimicrobial activity has emerged [5], rendered kefirs as probiotic products. Kefir starter cultures or so-called kefir grains are made of exopolysaccharides (EPSs) produced by some bacteria such as Lactobacillus hilgardii TMW 1.828 in water kefir from Germany [6], and L. kefirano- 
faciens [7]. Some Acetobacter sp. also can produce EPS such as Acetobacter tropicalis SKU1100 [8]; however, Acetobacter sp. from kefir has not been reported as an EPS producer. Microbial EPSs are polymers, primarily consisting of carbohydrates biosynthesized by bacteria [9], fungi [10] and cyanobacteria [11]. EPSs are grouped into two types as (1) Heteropolysaccharides with high molecular mass composed of numerous sugar monomers, synthesized by the orchestrated action of various glycosyl transferases, and (2) Homopolysaccharides with lower molecular mass consisting of one sugar monomer synthesized by one or few glycosyl transferases [12]. The antioxidant activities of these microbial EPSs are not clearly understood, however they have typically been attributed to the presence of excessive numbers of different monomers, high hydrophobicity and a strong anionic nature [13]. Although kefirs have existed in Thailand for some time, knowledge concerning their properties is scarce. Recently, the first report of Thai milk kefir from Kamphaeng Phet Province was documented [14] but Thai water kefir has yet to be studied. Also, knowledge of EPS producers in Thai water kefir is still limited. Thus, here the microbial strains of Thai water kefir from Nakhon Ratchasima Province, commonly homemade by local villagers, was determined using $16 \mathrm{~S}$ rRNA gene analysis and EPS producers were identified. The antioxidant and anti-tyrosinase activities of bacterial EPSs were also examined.

\section{Materials and Methods}

\section{Water kefir preparation}

A water kefir grain was purchased on-line from a supplier (JibKefirWater) in Nakhon Ratchasima Province, Thailand. This kefir grain is commonly used to produce water kefir by local villagers in the region. The water kefir grain was prepared in a sucrose solution (100 g/l) in $100 \mathrm{ml}$ sterile distilled water in a $250 \mathrm{ml}$ flask aerobically incubated at $37^{\circ} \mathrm{C}$ without shaking for 2 days. Kefir grain was propagated under laboratory conditions twice to eliminate the environmental effects from the supplier and the process was repeated to obtain water kefir.

\section{Growth conditions of microorganisms}

Microbes from water kefir were grown aerobically on three media including De Man, Rogosa and Sharpe (MRS) agar (HIMedia Laboratories, India) supplemented with bromocresol purple, glucose, yeast extract, calcium carbonate $(\mathrm{GYC})$ agar at $37^{\circ} \mathrm{C}$ and yeast extract peptone dextrose (YPD) agar (HIMedia Laboratories, India) at $30^{\circ} \mathrm{C}$ for selection of $\mathrm{LAB}, \mathrm{AAB}$ and yeasts, respectively as previously described [14]. Positive colonies were streaked five times on selective media to obtain pure isolates. Pure isolates were identified using PCR-based 16S rRNA and 26S rRNA gene analyses for bacteria and yeasts, respectively. All microbial isolates were deposited in the Natural Antioxidant Innovation Research Unit, Department of Biotechnology, Faculty of Technology, Mahasarakham University, Thailand (WDCM 1160).

\section{Microbial identification and phylogenetic tree construction}

Genomic DNA isolation from the selected microbes was performed as previously reported [14]. Bacteria were identified by partial sequencing of the $16 \mathrm{~S}$ rRNA amplified with the universal forward primer AmpF (5'GAGAGTTTGATYCTGGCTCAG-3') and the reverse primer AmpR (5'-AAGGAGGTGATCCARCCGCA -3'), and a PCR program of $94^{\circ} \mathrm{C}$ for $2 \mathrm{~min}, 32$ cycles of $94^{\circ} \mathrm{C}$ for $45 \mathrm{~s}, 54^{\circ} \mathrm{C}$ for $45 \mathrm{~s}, 72^{\circ} \mathrm{C}$ for $1 \mathrm{~min}$ and a last step at $72^{\circ} \mathrm{C}$ for $7 \mathrm{~min}$. Yeasts were identified by partial sequencing of the $26 \mathrm{~S}$ rRNA using the primers NL1 (5'GCATATCAATAAGCGGAGGAAAAG-3') and NL4 (5'GGTCCGTGTTTCAAGACG G-3'). The PCR reaction mixture $(25 \mu \mathrm{l})$ consisted of $0.1 \mathrm{mM}$ of each deoxynucleoside triphosphate, 0.75 U Taq polymerase (Vivantis, Malaysia), 5 pmol of each primer and $1 \mu \mathrm{g}$ of genomic DNA. The PCR products were resolved by electrophoresis in a $0.8 \%$ (w/v) agarose gel (Vivantis, Malaysia), cleaned-up using GF-1 PCR Clean-up kit (Vivantis, Malaysia) according to the manufacturer's instructions, and sent to $1^{\text {st }}$ Base Co. Ltd. (Malaysia) for sequencing. The identities of the isolates were determined on the basis of the highest matching score on BLAST search. Bootstrapped phylogenetic trees of all isolated bacteria based on 16S rRNA partial sequences and yeasts based on $26 \mathrm{~S}$ rRNA partial sequences were constructed using MEGA 7.0 software [15] with maximum likelihood method using Kimura 2-parameter [16] at 1,000 Bootstrap replications. The phylogenetic trees were drawn by FigTree [17]. 


\section{Screening for microbial EPS producers}

Screening was performed using the disk diffusion method [18]. Each identified isolate was cultured overnight in their corresponding broth till the culture reached $\mathrm{OD}_{600 \mathrm{~nm}} \sim 0.4$. Bacterial culture $(20 \mu \mathrm{l})$ was then pipetted on a sterile disk plated on modified MRS agar without glucose (for LAB and $\mathrm{AAB}$ ), and modified YPD agar without dextrose (for yeasts) containing $8 \%$ specific sugar (glucose, sucrose or lactose) to screen for EPS-producing capacity from identified $\mathrm{LAB}, \mathrm{AAB}$ and yeasts. Plates were aerobically incubated for 3 days at $37^{\circ} \mathrm{C}$ for bacteria and $30^{\circ} \mathrm{C}$ for yeasts. EPS producers were identified as having a slimy, ropy or glossy appearance and could be pulled up using an inoculating loop [14]. Negative controls included MRS medium with no bacteria, Lactobacillus sp. isolated from Thai Isan sausage and Escherichia coli DH5 $\alpha$ which do not produce EPS.

\section{Scavenging of DPPH free radical by bacterial superna- tants}

The 2,2-diphenyl-1-picrylhydrazyl (DPPH) radicalscavenging capacity of bacterial isolates was determined from their supernatants possibly containing EPS released from bacterial cells. LAB cultures $(10 \mathrm{ml})$ in modified MRS broth (without glucose) and AAB cultures (10 ml) in LB broth (pH 7.0) were grown on $8 \%$ sucrose till $\mathrm{OD}_{600 \mathrm{~nm}} \sim 0.7$ was reached, and then centrifuged at $16,100 \mathrm{~g}$ for $30 \mathrm{~min}$. Briefly, $1.0 \mathrm{ml}$ of bacterial supernatant was added to $2.0 \mathrm{ml}$ ethanolic DPPH radical solution $(0.05 \mathrm{mM})$. The mixture was mixed vigorously and incubated at room temperature in the dark for $30 \mathrm{~min}$. The controls included only broth containing $8 \%$ sucrose and DPPH solution whereas the blanks contained only ethanol. Absorbance of the resulting solution was measured in triplicate at $517 \mathrm{~nm}$. Scavenging ability of bacterial supernatant was defined as:

$$
\begin{aligned}
& \text { Scavenging activity }(\%) \\
& \quad=\left[1-\left(A_{\text {sample }}-A_{\text {blank }}\right) / A_{\text {control }}\right] \times 100
\end{aligned}
$$

\section{Extraction of crude EPS}

Each culture from the four selected bacteria with high DPPH scavenging activity from the above assay; $A$. pasteurianus WS3, A. pasteurianus WS6, P. pentosaceus WS11 and L. casei WS13 was collected at $72 \mathrm{~h}$ during a stationary phase $\left(\mathrm{OD}_{600 \mathrm{~nm}} \sim 0.7\right)$. The cultures were centrifuged at $16,100 \mathrm{~g}$ at $4^{\circ} \mathrm{C}$ for $30 \mathrm{~min}$, and then two volumes of cold absolute ethanol were added to the supernatant and stored at $4^{\circ} \mathrm{C}$ for $24 \mathrm{~h}$. After centrifugation, the EPS precipitate was washed twice with absolute ethanol. The fresh cell pellet was weighed before drying to constant weight in an oven at $60^{\circ} \mathrm{C}$. Dried EPS (200 mg) was dissolved in $10 \mathrm{ml}$ sterile distilled water, and $20 \%(\mathrm{v} / \mathrm{v})$ trichloroacetic acid was added to remove protein contamination as a precipitate at $4^{\circ} \mathrm{C}$ for $1 \mathrm{~h}$. The mixture was then centrifuged at $16,100 \mathrm{~g}$ for $10 \mathrm{~min}$. The supernatant (EPS fraction) was partially purified using Vivaspin $10 \mathrm{kDa}$ MWCO membrane ultrafiltration (Sigma-Aldrich, Singapore) and freeze dried. The dried crude EPS was weighed and kept at $-20^{\circ} \mathrm{C}$ until required.

\section{Ferric reducing antioxidant power (FRAP) assay}

The antioxidant activity of each bacterial EPS was evaluated using FRAP assay with some modifications to the previous method [19]. FRAP reagent was prepared in $300 \mathrm{mM}$ acetate buffer $\mathrm{pH} 3.6$ by adding acetic acid, $10 \mathrm{mM}$ 2,4,6-Tris(2-pyridyl)-s-triazine (TPTZ) solution in $40 \mathrm{mM} \mathrm{HCl}$ and $20 \mathrm{mM}$ iron (III) chloride solution $\left(\mathrm{FeCl}_{3}\right)$ at proportions of $1: 1: 10(\mathrm{v} / \mathrm{v} / \mathrm{v})$, respectively. A total of $100 \mu \mathrm{l}$ EPS solution $(20 \mathrm{mg} / \mathrm{ml})$ was added to $3,000 \mu \mathrm{l}$ of the FRAP reagent, mixed well and incubated for $15 \mathrm{~min}$ at room temperature. Absorbance was measured at $595 \mathrm{~nm}$ using a spectrophotometer with iron (II) sulfate $\left(\mathrm{FeSO}_{4}\right)$ used as a standard. Results were expressed as $\mathrm{mM} \mathrm{FeSO}_{4} / \mathrm{g} \mathrm{DW}$.

\section{ABTS $^{++}$antioxidant assay}

The ABTS $^{\cdot+}$ antioxidant assay was carried out with some modifications to the previously reported method [20]. A total of $100 \mu \mathrm{l}$ EPS sample $(20 \mathrm{mg} / \mathrm{ml})$ was added to $1,000 \mu \mathrm{l}$ of 2,2'-Azino-di(3-ethyl-benzthiazoline sulfonic acid $\left(\mathrm{ABTS}^{\circ+}\right)$ radical cation solution and mixed well. Absorbance was measured at $734 \mathrm{~nm}$ after $15 \mathrm{~min}$ incubation at room temperature. The control included only water and $\mathrm{ABTS}^{\cdot+}$ solution whereas the blank contained only water. The antioxidant ability of bacterial EPS was defined as:

$$
\begin{aligned}
& \text { ABTS }^{\cdot+} \text { antioxidant activity }(\%) \\
& \quad=\left[1-\left(A_{\text {sample }}-A_{\text {blank }}\right) / A_{\text {control }}\right] \times 100
\end{aligned}
$$




\section{Anti-tyrosinase activity of EPS}

This was performed according to a previous report [21]. Ten microliters of EPS extract $(20 \mathrm{mg} / \mathrm{ml})$ was mixed with $20 \mu \mathrm{l}$ of $1000 \mathrm{U} / \mathrm{ml}$ mushroom tyrosinase (Sigma-Aldrich, Singapore) in $110 \mu$ l of phosphate buffer $\mathrm{pH} 6.5$ in a microplate, and incubated at $37^{\circ} \mathrm{C}$ for $10 \mathrm{~min}$ at room temperature. Ten microliters of $10 \mathrm{mM}$ levodopa (L-DOPA) was added to the mixture and incubated at $37^{\circ} \mathrm{C}$ for $40 \mathrm{~min}$ at room temperature. Absorbance at $490 \mathrm{~nm}$ was recorded using a spectrophotometer with a buffer mixed with L-DOPA and tyrosinase as a control. All experiments were performed in triplicate. Tyrosinase inhibition was calculated from the equation below:

$$
\begin{aligned}
& \text { Tyrosinase inhibition }(\%) \\
& \quad=\left[1-\left(\mathrm{A}_{490 \mathrm{~nm}} \text { sample }\right) /\left(\mathrm{A}_{490 \mathrm{~nm}} \text { control }\right)\right] \times 100
\end{aligned}
$$

\section{Scanning electron microscopic analysis}

A scanning electron microscope (SEM) was used to study the surface morphology of Thai water kefir grain and crude EPS extract. Dried water kefir or EPS (2 mg) was coated with a $30 \mathrm{~nm}$ layer of gold before observation under SEM (Carl Zeiss, Leo/1450) at accelerating voltage of $5 \mathrm{kV}$ at the Faculty of Engineering, Khon Kaen University, Thailand.

\section{Statistical analysis of data}

Measurements were obtained in triplicate as means \pm standard deviation (SD). Statistical analysis was performed using one-way analysis of variance (ANOVA) and Tukey's honest significant difference (HDS) test by SPSS software (version 19) at $p<0.05$.
Table 1. Microbial enumeration on three media.

\begin{tabular}{ccc}
\hline $\begin{array}{c}\text { Medium/ } \\
\text { Microbes }\end{array}$ & $\begin{array}{c}\text { Microbial enumeration } \\
(\mathrm{CFU} / \mathrm{ml})\end{array}$ & $\begin{array}{c}\text { Selected colonies/ } \\
\text { total colonies }\end{array}$ \\
\hline MRS/LAB & $6.2 \times 10^{8}$ & $31 / 62$ \\
GYC/AAB & $6.2 \times 10^{6}$ & $31 / 62$ \\
YPD/Yeast & $1.0 \times 10^{8}$ & $50 / 100$ \\
\hline
\end{tabular}

\section{Results}

\section{Microbial strains in Thai water kefir}

The first report of microbial consortia in Thai milk kefir has recently been published [14], however microbes in Thai water kefir have yet to be elucidated. Thus the aim of this study was to determine the microbial strains in Thai water kefir. Thai water kefir grain appeared clear, light brown-orange and minute (Fig. 1A) from Nakhon Ratchasima Province (Fig. 1B) [1458'29.99" N $\left.102^{\circ} 05^{\prime} 60.00^{\prime \prime} \mathrm{E}\right]$ and contained Gram-positive rod, coccal, and Gram-negative rod bacteria (Fig. 1C) and yeasts (Fig. 1D). Sixty-two colonies $\left(6.2 \times 10^{5} \mathrm{CFU} / \mathrm{ml}\right.$ from $10^{-4}$ dilution) were grown on MRS agar supplemented with bromocresol purple (Table 1) with yellow zone indication of lactic acid production by putative LAB after 3 days of incubation at $37^{\circ} \mathrm{C}$, and 62 colonies $\left(6.2 \times 10^{8} \mathrm{CFU} / \mathrm{ml}\right.$ from $10^{-6}$ dilution) were grown on GYC agar with a clear zone indicating acetic acid production by putative $\mathrm{AAB}$ that cleared out calcium carbonate. One hundred yeast colonies $\left(1.0 \times 10^{8} \mathrm{CFU} / \mathrm{ml}\right.$ from $10^{-5}$ dilution) were grown on YPD agar at $30^{\circ} \mathrm{C}$. Half the colonies on each plate were then selected, based on different colony morphology and Gram stained, then streaked through 5 pas-
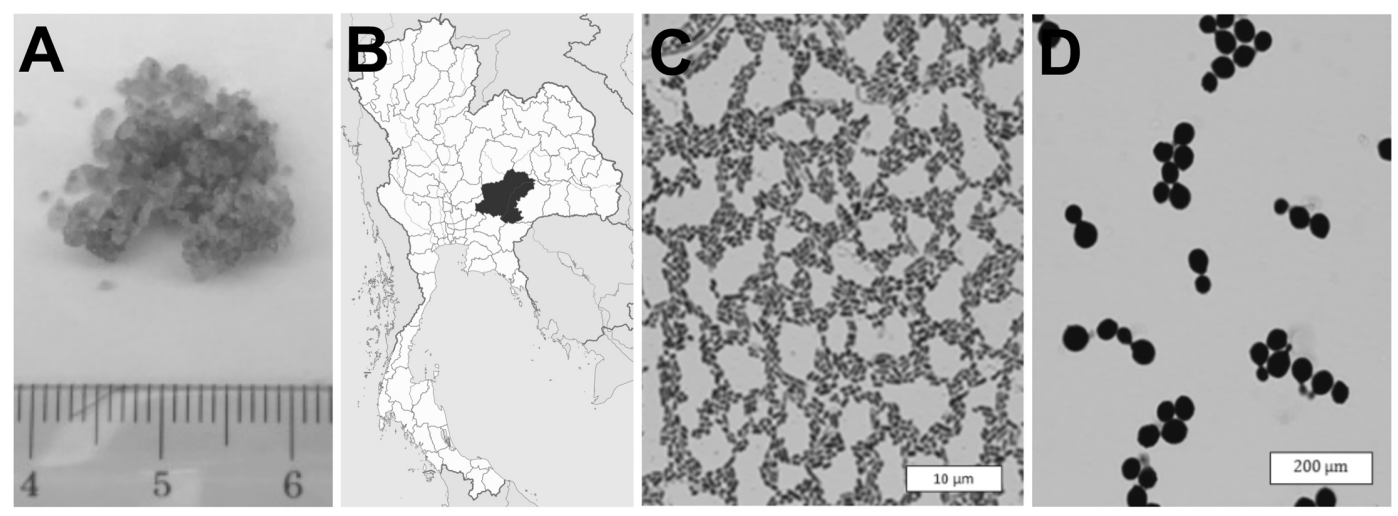

Fig. 1. Thai water kefir. (A) Size of water kefir grain. (B) Location of Nakhon Ratchasima Province in Thailand. (C) Magnification at $1000 \mathrm{X}$ of $L A B$ in water kefir under microscope. (D) Magnification at 1000X of yeasts in water kefir under microscope. 
Table 2. Eighteen strains identified out of 62 bacterial isolates and 15 strains identified out of 50 yeast isolates from Thai water kefir.

\begin{tabular}{|c|c|c|c|c|c|}
\hline \multirow{2}{*}{ No. } & \multirow{2}{*}{$\frac{\text { Bacterial strains }}{\text { Acetobacter pasteurianus WS1 }}$} & \multicolumn{3}{|c|}{ Isolates (\%) $)^{a}$ Accession no. ${ }^{b} \%$ identity $^{c}$} & \multirow{2}{*}{$\begin{array}{l}\text { Origin of closest relative }{ }^{\mathrm{d}} \\
\text { MF179549.1 Acetobacter pasteurianus L1, fermented tofu } \\
\text { whey, China }\end{array}$} \\
\hline & & $13(21.31 \%)$ & LC336429.1 & $99 \%$ & \\
\hline 2 & Acetobacter pasteurianus WS2 & $12(19.67 \%)$ & LC336430.1 & $99 \%$ & $\begin{array}{l}\text { KX622697.1 Acetobacter pasteurianus JBARES-MG-3, } \\
\text { makgeolli (Korean fermentation alcohol, Korea }\end{array}$ \\
\hline 3 & Acetobacter pasteurianus WS3 & $7(11.48 \%)$ & LC336431.1 & $97 \%$ & $\begin{array}{l}\text { KY287771.1 Acetobacter pasteurianus DI10SB1, } \\
\text { fermentation of Mezcal in Oaxaca, Mexico }\end{array}$ \\
\hline 4 & Acetobacter pasteurianus WS4 & $7(11.48 \%)$ & LC336432.1 & $87 \%$ & $\begin{array}{l}\text { KT283052.1 Acetobacter pasteurianus AS1.41, hinese } \\
\text { vinegar Pei, China }\end{array}$ \\
\hline 5 & Acetobacter pasteurianus WS5 & $4(6.56 \%)$ & LC336433.1 & $98 \%$ & KX424632.1 Acetobacter pasteurianus 1HSc, palm sap, Italy \\
\hline 6 & Acetobacter pasteurianus WS6 & $1(1.64 \%)$ & LC336434.1 & $100 \%$ & $\begin{array}{l}\text { AB906418.1 Acetobacter pasteurianus SKU129, santol, } \\
\text { Thailand }\end{array}$ \\
\hline 7 & Acetobacter pasteurianus WS7 & $1(1.64 \%)$ & LC336435.1 & $98 \%$ & KX424631.1 Acetobacter pasteurianus 1RMc, palm sap, Italy \\
\hline 8 & Acetobacter sp. WS8 & $1(1.64 \%)$ & LC336436.1 & $96 \%$ & $\begin{array}{l}\text { KP205437.1 Acetobacter sp. SLV-203, Korean traditional } \\
\text { vinegar, Korea }\end{array}$ \\
\hline 9 & Acetobacter tropicalis WS9 & $1(1.64 \%)$ & LC336437.1 & $99 \%$ & $\begin{array}{l}\text { MG225384.1 Acetobacter tropicalis LAABBM7A, fermented } \\
\text { beans, Brazil }\end{array}$ \\
\hline 10 & Pediococcus pentosaceus WS10 & $4(6.56 \%)$ & LC336438.1 & $98 \%$ & $\begin{array}{l}\text { KU892402.1 Pediococcus pentosaceus PPOF134, traditional } \\
\text { cereal-based fermented food, Nigeria }\end{array}$ \\
\hline 11 & Pediococcus pentosaceus WS11 & $1(1.64 \%)$ & LC336439.1 & $98 \%$ & $\begin{array}{l}\text { KP189225.1 Pediococcus pentosaceus V3-100, fermentation } \\
\text { of wheat for lemzeiet production, Algeria }\end{array}$ \\
\hline 12 & Pediococcus pentosaceus WS12 & $1(1.64 \%)$ & LC336440.1 & $94 \%$ & $\begin{array}{l}\text { JQ446485.1 Pediococcus pentosaceus QN1D, traditional } \\
\text { fermented foods, Ecuador }\end{array}$ \\
\hline 13 & Lactobacillus casei WS13 & $3(4.92 \%)$ & LC336441.1 & $100 \%$ & MF429431.1 Lactobacillus casei CAU2903, rushan, China \\
\hline 14 & Lactobacillus casei WS14 & $1(1.64 \%)$ & LC336442.1 & $99 \%$ & MF354567.1 Lactobacillus casei CAU:2544, soil, China \\
\hline 15 & Lactobacillus casei WS15 & $1(1.64 \%)$ & LC336443.1 & $99 \%$ & KM485569.1 Lactobacillus casei MJM60422, food, Korea \\
\hline 16 & Lactococcus hircilactis WS16 & $1(1.64 \%)$ & LC336444.1 & $100 \%$ & KJ201026.1 Lactococcus hircilactis DSM 28960, milk, Italy \\
\hline 17 & Lactococcus lactis WS17 & $1(1.64 \%)$ & LC336445.1 & $98 \%$ & $\begin{array}{l}\text { HM218132.1 Lactococcus lactis subsp. lactis NM26-6, } \\
\text { naturally fermented dairy products, China }\end{array}$ \\
\hline 18 & Lactococcus lactis WS18 & $1(1.64 \%)$ & LC336446.1 & $98 \%$ & KT260999.1, Lactococcus lactis RCB787, bat guano, India \\
\hline & Total bacteria & $62(100 \%)$ & & & \\
\hline
\end{tabular}

sages to obtain purified isolates. Some colonies grown on GYC agar were identified as LAB and some on MRS agar were identified as AAB. A BLAST search determined that 9 strains of Acetobacter spp. were predominant as well as 9 strains of LAB including Lactobaciilus spp., Lactococcus spp. and Pediococcus spp. with 13 yeast strains as mainly Saccharomyces spp. and 2 strains of Meyerozyma guilliermondii (Table 2). Most of these bacteria shared high similarity (98-100\%) with closest relatives found in Asian countries including China, Thailand, Korea and India. Most S. cerevisiae strains are extremely similar (99-100\% identity) with closest relatives found in China. $M$. guilliermondii found in our work was similar (98\% identity) to yeasts isolated from cultivated kiwi fruit land and fruit skin in China. Phylogenetic analysis of bacteria (Fig. 2A) showed two main clusters of bacteria classified into LAB and AAB. L. hircilactis WS16, as an outlier, was evolutionarily different from other $\mathrm{LAB}$ and $\mathrm{AAB}$. $\mathrm{LAB}$ can be grouped into 3 sub-groups as L. casei, Pc. pentosaceus and L. lactis. The reference strain $L$. casei KLDS 1.0720 (EU626005.1) was evolutionarily similar to our L. casei strains. However, another reference strain, L. lactis NS32 (EU194346.1) found in Brazilian kefir [4] was evo- 
Table 2. Continued.

\begin{tabular}{|c|c|c|c|c|c|}
\hline No. & Yeast strains & Isolates (\%) ${ }^{\mathrm{a}}$ & Accession no. ${ }^{b}$ & $\%$ identity $^{c}$ & Origin of closest relative $^{d}$ \\
\hline 19 & Saccharomyces cerevisiae TC1 & $10(20 \%)$ & LC336447.1 & $100 \%$ & EU556339.1 Saccharomyces cerevisiae, China \\
\hline 20 & Saccharomyces cerevisiae TC2 & $9(18 \%)$ & LC336448.1 & $99 \%$ & $\begin{array}{l}\text { KJ850219.1 Saccharomyces cerevisiae L4, distiller's yeast, } \\
\text { China }\end{array}$ \\
\hline 21 & Saccharomyces cerevisiae TC3 & $8(16 \%)$ & LC336449.1 & $99 \%$ & $\begin{array}{l}\text { KJ850221.1 Saccharomyces cerevisiae L6, distller's yeast, } \\
\text { China }\end{array}$ \\
\hline 22 & Saccharomyces cerevisiae TC4 & $5(10 \%)$ & LC336450.1 & $100 \%$ & KT922993.1 Saccharomyces cerevisiae NS-G-24, wine, Spain \\
\hline 23 & Saccharomyces cerevisiae TC5 & $3(6 \%)$ & LC336451.1 & $100 \%$ & $\begin{array}{l}\text { JQ824874.1 Saccharomyces cerevisiae LCBG-3Y4, sponta- } \\
\text { neous fermentation of mescal, Mexico }\end{array}$ \\
\hline 24 & Saccharomyces cerevisiae TC6 & $2(4 \%)$ & LC336452.1 & $99 \%$ & $\begin{array}{l}\text { HM107783.1 Saccharomyces cerevisiae q5, Chinese rice } \\
\text { wine, China }\end{array}$ \\
\hline 25 & Saccharomyces cerevisiae TC7 & $2(4 \%)$ & LC336453.1 & $99 \%$ & $\begin{array}{l}\text { HM107797.1 Saccharomyces cerevisiae 4, Chinese rice } \\
\text { wine, China }\end{array}$ \\
\hline 26 & Saccharomyces cerevisiae TC8 & $2(4 \%)$ & LC336454.1 & $100 \%$ & $\begin{array}{l}\text { JQ824873.1 Saccharomyces cerevisiae LCBG-3Y8, sponta- } \\
\text { neous fermentation of mescal, Mexico }\end{array}$ \\
\hline 27 & Saccharomyces cerevisiae TC9 & $2(4 \%)$ & LC336455.1 & $100 \%$ & $\begin{array}{l}\text { HM191635.1 Saccharomyces cerevisiae NL4, Musalais } \\
\text { grape wine, China }\end{array}$ \\
\hline 28 & Saccharomyces cerevisiae TC10 & $2(4 \%)$ & LC336456.1 & $100 \%$ & $\begin{array}{l}\text { HM191636.1 Saccharomyces cerevisiae NL5, Musalais } \\
\text { grape wine, China }\end{array}$ \\
\hline 29 & Saccharomyces cerevisiae TC11 & $1(2 \%)$ & LC336457.1 & $100 \%$ & JQ512832.1 Saccharomyces cerevisiae 29, distillery, Spain \\
\hline 30 & Saccharomyces cerevisiae TC12 & $1(2 \%)$ & LC336458.1 & $100 \%$ & $\begin{array}{l}\text { HM191649.1 Saccharomyces cerevisiae NL18, Musalais } \\
\text { grape wine, China }\end{array}$ \\
\hline 31 & Saccharomyces cerevisiae TC13 & $1(2 \%)$ & LC336808.1 & $100 \%$ & $\begin{array}{l}\text { HM123751.1 Saccharomyces cerevisiae SL7-5-2, fermenta- } \\
\text { tion solution, China }\end{array}$ \\
\hline 32 & Meyerozyma guilliermondii TC14 & $1(2 \%)$ & LC336809.1 & $99 \%$ & $\begin{array}{l}\text { KJ794675.1 Meyerozyma guilliermondii B-NC-13-OZ01, } \\
\text { kiwi fruit producing area, China }\end{array}$ \\
\hline 33 & Meyerozyma guilliermondii TC15 & $1(2 \%)$ & LC336810.1 & $99 \%$ & $\begin{array}{l}\text { JX041892.1 Meyerozyma guilliermondii DSSL-1, surface of } \\
\text { fruits, China }\end{array}$ \\
\hline
\end{tabular}

${ }^{a}$ Number of isolates and abundance percentage (\%) $=$ (identified number of isolates/total isolates) $\times 100$

${ }^{b}$ GenBank accession no. of strains on NCBI website (http://www.ncbi.nlm.nih.gov/pubmed)

'Based on BLAST search results, identity (\%) of strains compared to the closest relatives.

${ }^{\mathrm{d}}$ Based on BLAST search results, origin of the closest relatives.

lutionarily diverged from our Lactobacillus and Pediococcus strains. $\mathrm{AAB}$ can be grouped into 2 sub-groups as A. tropicalis and A. pasteurianus. A. tropicalis WS9 isolated from this study appeared to be evolutionarily similar to A. tropicalis NRIC 0312 (AB032354.1) in Belgian kefir [22], but different from A. orientalis (AB052707) found in Brazilian kefir [4]. The reference strain A. pasteurianus subsp. pasteurianus (X71863.1) found in Belgian kefir [22] was evolutionarily similar to our Acetobacter sp. WS1, A. pasteurianus WS5 and WS6. In addition, the phylogenetic analysis of yeasts (Fig. 2B) showed two main clusters of yeasts classified into S. cer- evisiae and $M$. guilliermondii. All of our S. cerevisiae strains from this work were evolutionarily similar to the two reference yeast strains, $S$. cerevisiae VI2 (EU441887.1) found in white wine in Italy and S. cerevisiae CHFY0901 (EU649673.1) from bioethanol production in South Korea. Another reference strain, $S$. cerevisiae (EU019225.1) isolated from Koumiss in Xinjiang of China seemed to be evolutionarily different from other Saccharomyces; however similar to Pichia guilliermondii HK58-2 (EF197951.1). The two isolates of $M$. guilliermondii (TC14 and TC15) are in a separate group from $S$. cerevisiae and $P$. guilliermondii indicating that 
A

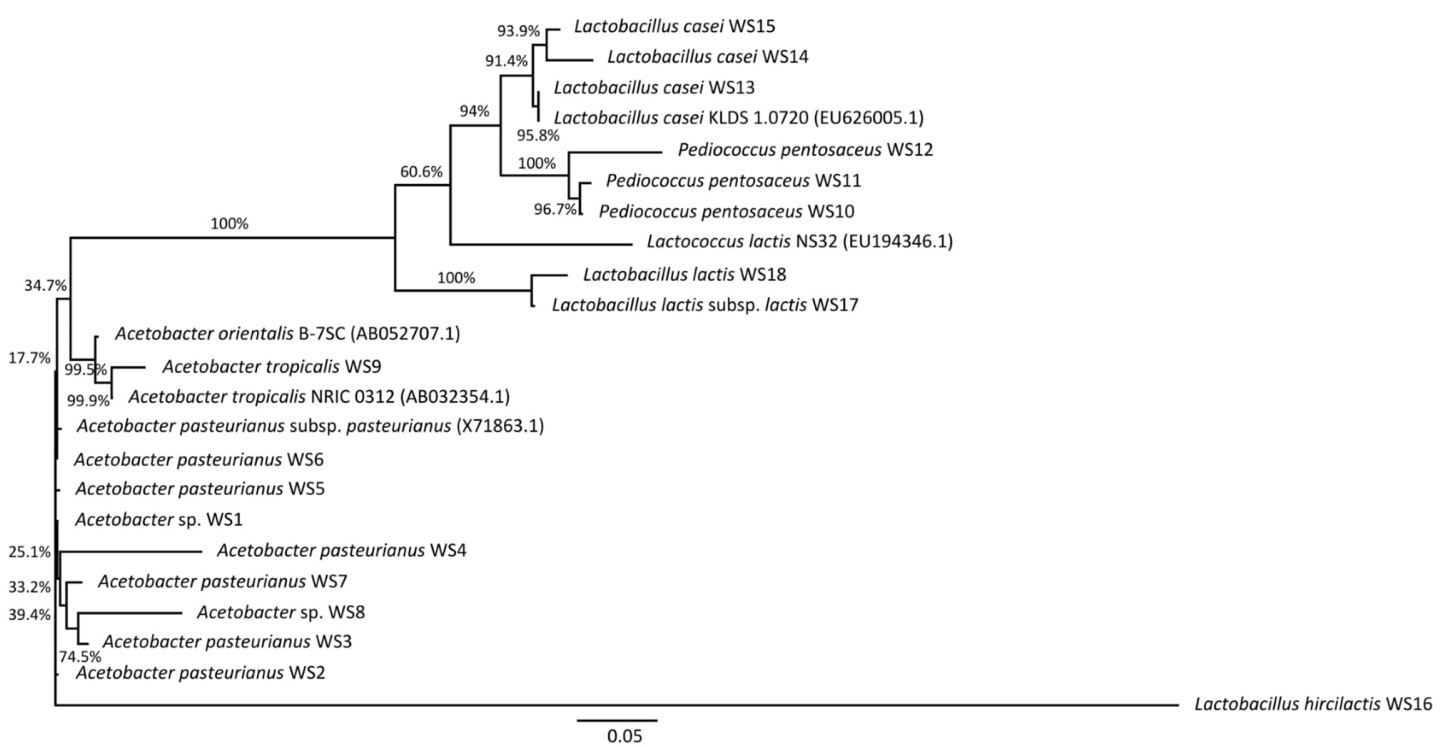

B

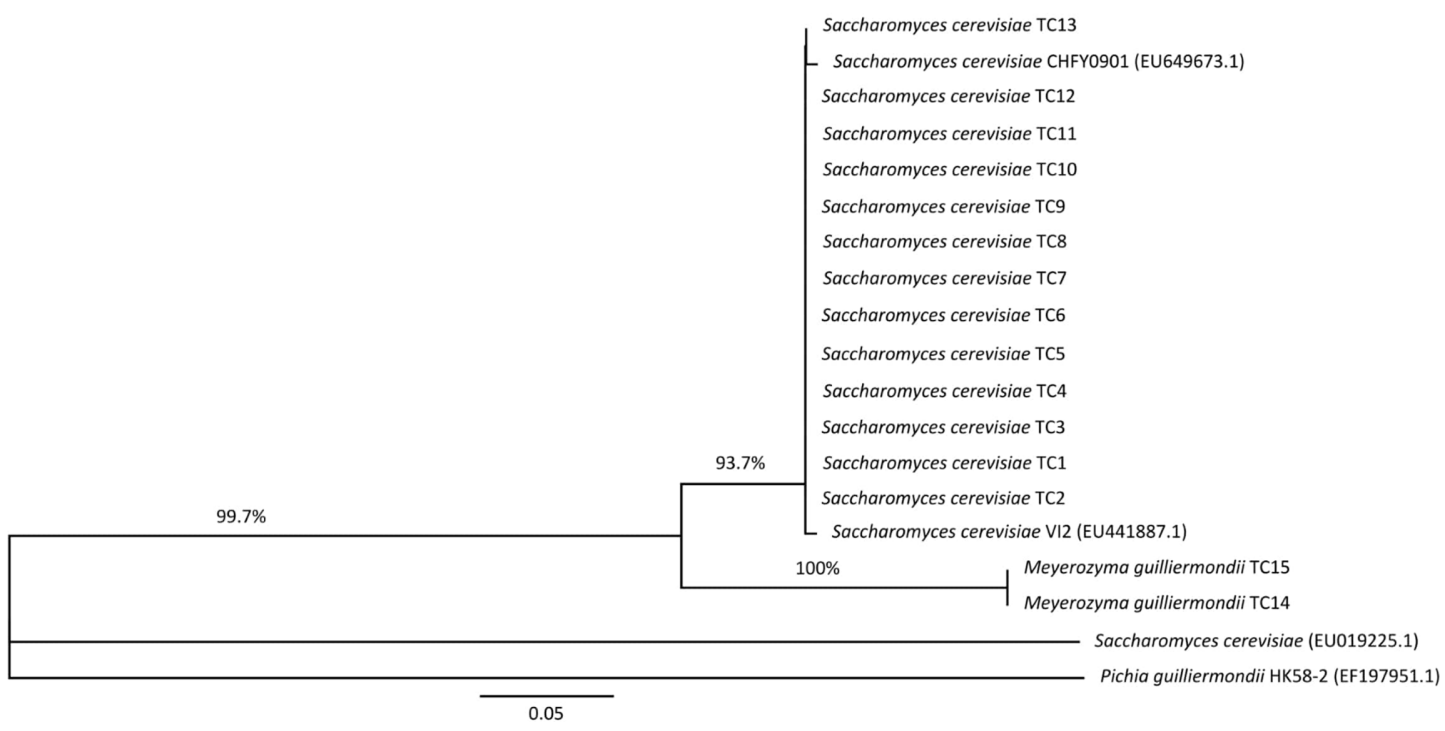

Fig. 2. Phylogenetic trees of microbes. (A) It was constructed from bacterial $16 \mathrm{~S}$ rRNA partial sequences (ca. 1,500 bp). (B) It was constructed from yeast 26S rRNA partial sequences (ca. $600 \mathrm{bp}$ ) based on Kimura 2-parameter using Maximum Likelihood method with 1,000 Bootstrap replications MEGA7. The phylogenetic trees were drawn using FigTree. The horizontal bar at the bottom of the figure represents a distance of 0.1 substitutions per site.

they were evolutionarily different.

\section{Bacterial EPS producers on sugars}

Since little was known about microbes responsible for EPS production in kefir grains, our aim was to identify EPS producers in Thai water kefir based on the disk diffusion method using 3 different sugars (80 g/l glucose, sucrose or lactose separately) in modified MRS agar without glucose (for bacteria) and modified YPD agar without dextrose (for yeasts). Many authors have reported on EPS-producing strains isolated from Tibetan kefir, with most identified as LAB such as Leuconostoc pseudomesenteroides [23] and L. plantarum [24]. Here, all LAB and $\mathrm{AAB}$ strains isolated in this study were found to be EPS producers, except when lactose was used. However, some bacterial strains including A. pasteurianus WS4, WS6, SW7, L. casei WS15 and L. lactis WS18 were unable to produce EPS on lactose 
Negative control E. coli DH5a
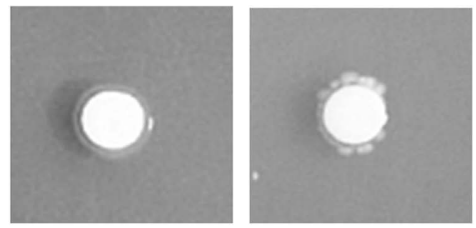

1) Acetobacter sp. WS1
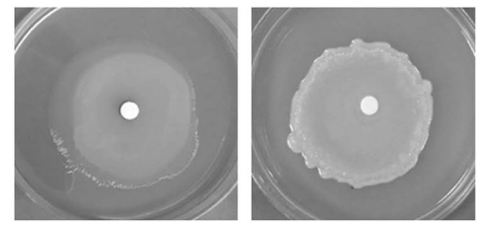

3) Acetobacter pasteurianus WS3
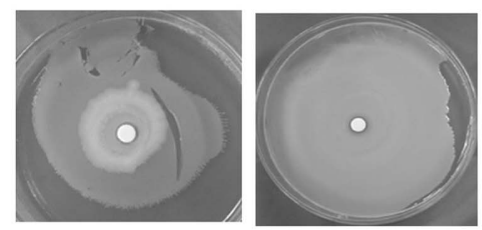

5) Acetobacter pasteurianus WS5
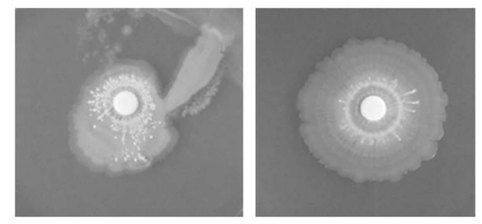

7) Acetobacter pasteurianus WS7
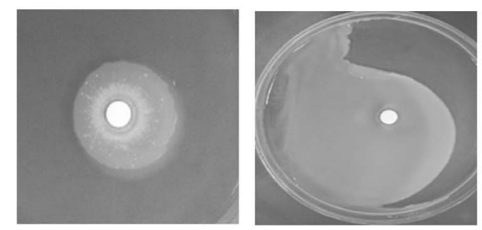

9) Acetobacter tropicalis WS9
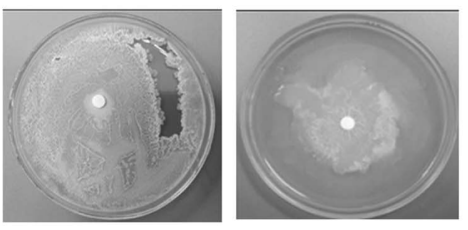

11) Pediococcus pentosaceus WS11
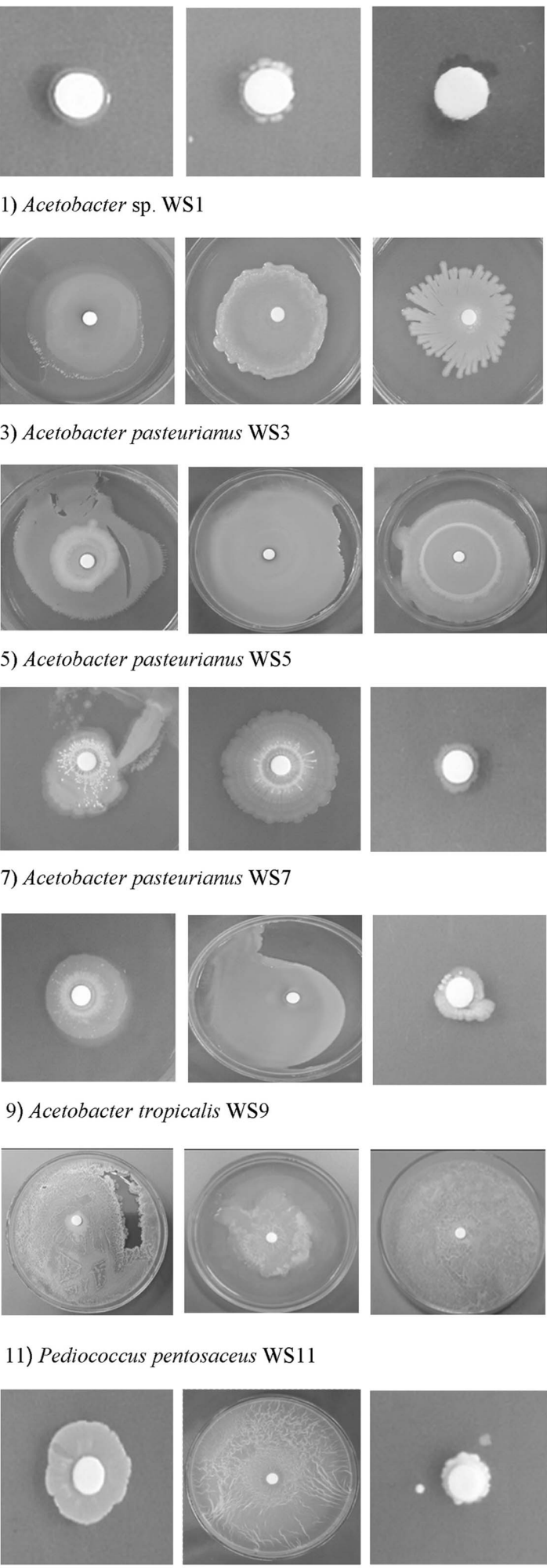

Negative control Lactobacillus sp.
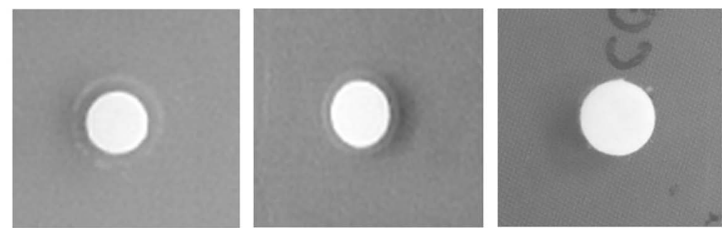

2) Acetobacter pasteurianus WS2
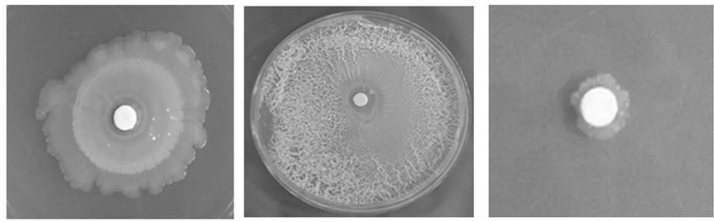

4) Acetobacter pasteurianus WS4
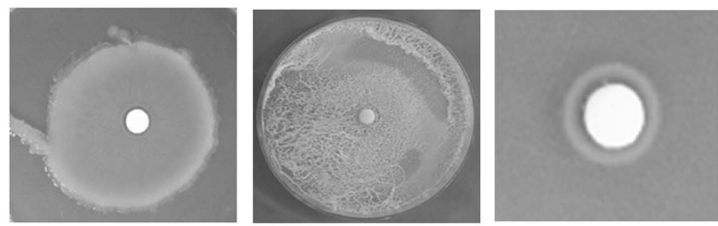

6) Acetobacter pasteurianus WS6
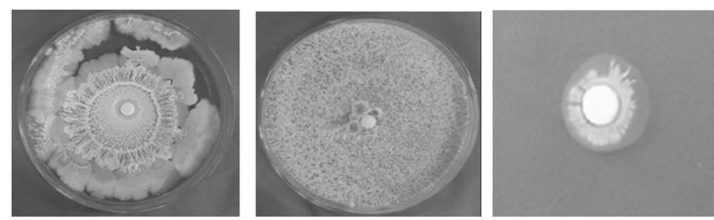

8) Acetobacter sp. WS8
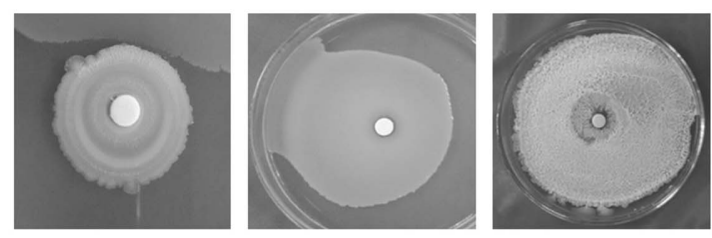

10) Pediococcus pentosaceus WS10
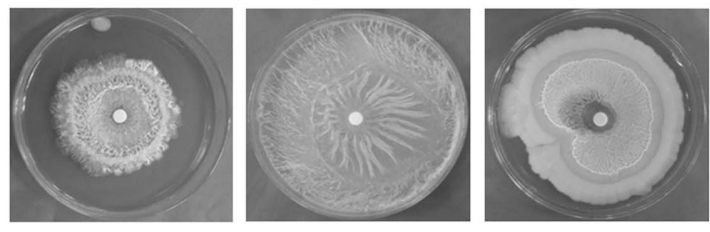

12) Pediococcus pentosaceus WS12
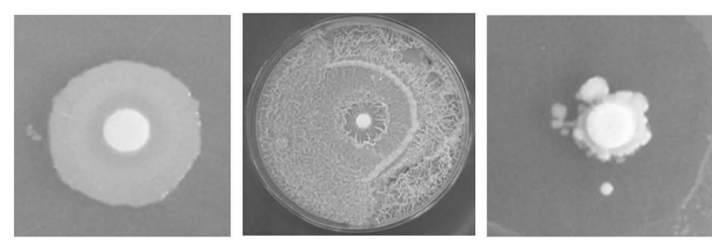

Fig. 3. EPS production from microbes. $A A B$ and $L A B$ were grown on $80 \mathrm{~g} / \mathrm{l}$ glucose (the first column), sucrose (the middle column) and lactose (the third column) containing agar after 3 days of incubation at $37^{\circ} \mathrm{C}$. 
13) Lactobacillus casei strain WS13
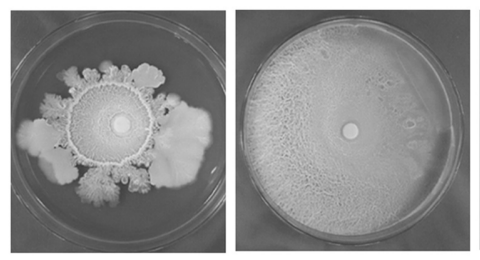

15) Lactobacillus casei strain WS15
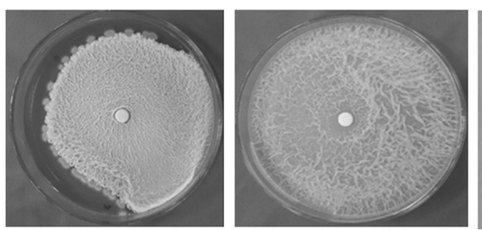

17) Lactococcus lactis subsp. lactis WS17
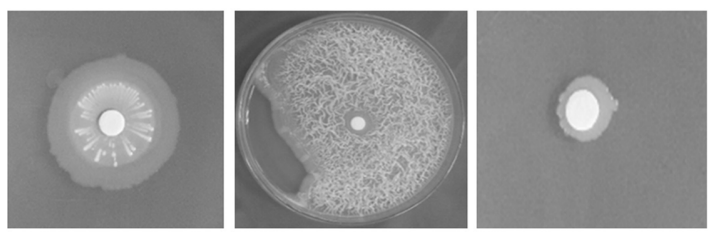

Fig. 3. Continued.

(Fig. 3). Different EPS appearances from different sugars were observed in the same bacteria, while diverse EPS appearances from the same sugar were observed in different bacteria (Fig. 3).

\section{Antioxidant and anti-tyrosinase activities of bacterial EPSs}

Antioxidant activities of all bacterial supernatants containing EPS were assessed using the DPPH scavenging assay. The three highest DPPH scavenging activities were observed in A. pasteurianus WS3, WS6 and WS1 supernatants at 64, 64 and $62 \%$, respectively, whilst the lowest activity was exhibited by $L$. hircilactis WS16 at $31 \%$ (Fig. 4). Most AAB and LAB showed 35-50\% scavenging activity. The four representative isolates with highest DPPH scavenging activity from each genus (Fig. 4) including A. pasteurianus WS3, A. pasteurianus WS6, Pc. pentosaceus WS11 and L. casei WS13 were selected for EPS production and determination of antioxidant and anti-tyrosinase activities of crude EPSs in further experiments. Crude EPS extracts from 4 bacteria were produced from 8\% sucrose in LB media ( $\mathrm{pH} 7.0$ ) for $72 \mathrm{~h}$. A. pasteurianus WS3 produced the highest EPS concentration of $1,505 \mathrm{mg}$ DW/L followed by A. pasteurianus
14) Lactobacillus casei strain WS14
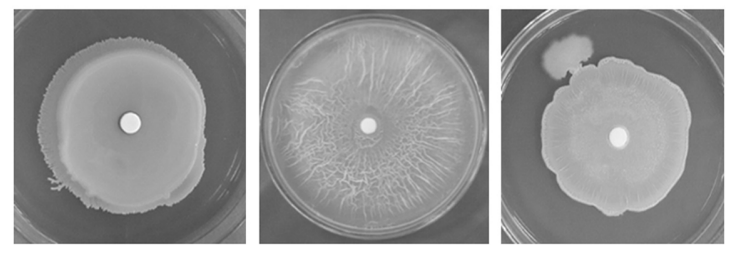

16) Lactococcus hircilactis WS16
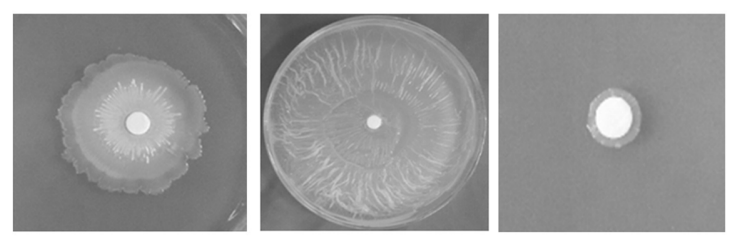

18) Lactococcus lactis WS18
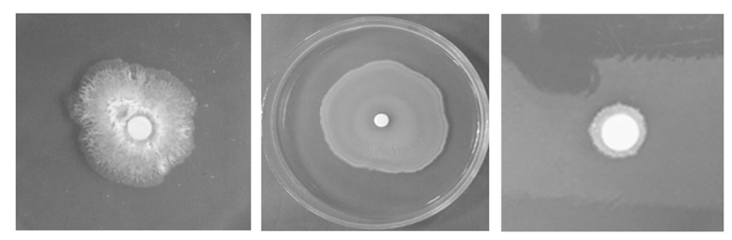

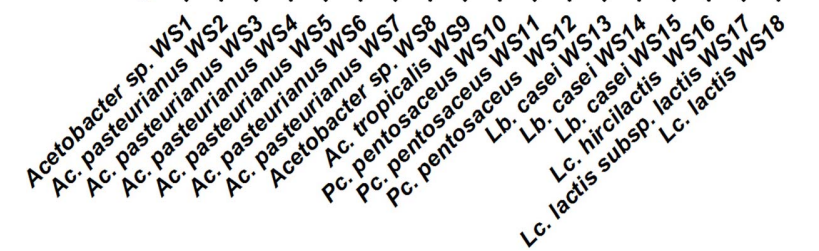

Fig. 4. DPPH scavenging activity (\%) of supernatants of eighteen EPS-producing bacteria isolated from Thai water kefir grown on $\mathbf{8 \%}$ sucrose for $\mathbf{2 4} \mathbf{h}$. Statistical significance $(p<0.05)$ was indicated by different letters using Tukey's HSD test.

WS6 (336 mg DW/L), L. casei WS13 (225 mg DW/L) and Pc. pentosaceus WS11 (176 mg DW/L) (Table 3). Reductive ability of EPS as an antioxidant was measured by 
Table 3. Dry weight of EPS, its antioxidant and anti-tyrosinase activity.

\begin{tabular}{lcccr}
\hline \multicolumn{1}{c}{ Bacterial EPS } & $\begin{array}{c}\text { Crude EPS } \\
\text { (mg DW/L) }\end{array}$ & $\begin{array}{c}\text { FRAP } \\
\left(\mathrm{FeSO}_{4} \mathrm{mM} / \mathrm{g}\right)\end{array}$ & $\begin{array}{c}\text { ABTS }^{+} \\
\text {(\% antioxidant activity) }\end{array}$ & $\begin{array}{c}\text { Tyrosinase inhibition } \\
(\%)\end{array}$ \\
\hline Acetobacter pasteurianus WS3 & $1,505 \pm 3$ & $280 \pm 31$ & $87.83 \pm 0.71$ & $20.35 \pm 2.52$ \\
Acetobacter pasteurianus WS6 & $336 \pm 10$ & $141 \pm 24$ & $86.12 \pm 0.67$ & $8.17 \pm 0.31$ \\
Pediococcus pentosaceus WS11 & $176 \pm 8$ & $132 \pm 8$ & $74.73 \pm 1.32$ & $3.89 \pm 1.27$ \\
Lactobacillus casei WS13 & $225 \pm 5$ & $150 \pm 19$ & $92.40 \pm 0.36$ & $12.56 \pm 1.12$ \\
\hline
\end{tabular}

${ }^{a}$ Crude EPSs were obtained from bacterial cultures in $8 \%$ sucrose-containing media $(1 \mathrm{~L})$ for $72 \mathrm{~h}$. Letters indicate significant differences between values in the columns $(p<0.05)$ using Tukey's HSD test.

investigating the $\mathrm{Fe}^{3+}-\mathrm{Fe}^{2+}$ transformation in FRAP assay in the presence of TPTZ forming an intense blue $\mathrm{Fe}^{2+}$-TPTZ complex with an absorption maximum at 595 nm [19]. A. pasteurianus WS3 EPS exhibited the highest FRAP value of $280 \mathrm{mM} \mathrm{FeSO}_{4} / \mathrm{g}$ which was insignificantly different from those of the other 3 bacterial EPSs ranging from 132-150 mM FeSO $4 / \mathrm{g}$ (Table 3). However, ABTS assay results showed that L. casei WS13 EPS exhibited significantly higher antioxidant capacity of 92.49\% than EPSs from A. pasteurianus WS3 and WS6 (87.83\% and $86.12 \%$, respectively). A. pasteurianus WS3 EPS showed the highest anti-tyrosinase inhibition activity at $20.35 \%$ with significant difference among 4 bacterial EPSs (Table 3).

\section{SEM analysis}

The SEM micrographs showed that water kefir grains had ovoid structures of the predominant Acetobacter spp. accounting for $77.06 \%$ abundance among the bacterial population adhered to each other on the grainy and uneven surface covered with pores (Fig. 5A) presumably offering water-holding capacity [25]. The EPS surface morphology of A. pasteurianus WS3 showed a smooth thin film (Fig. 5B).
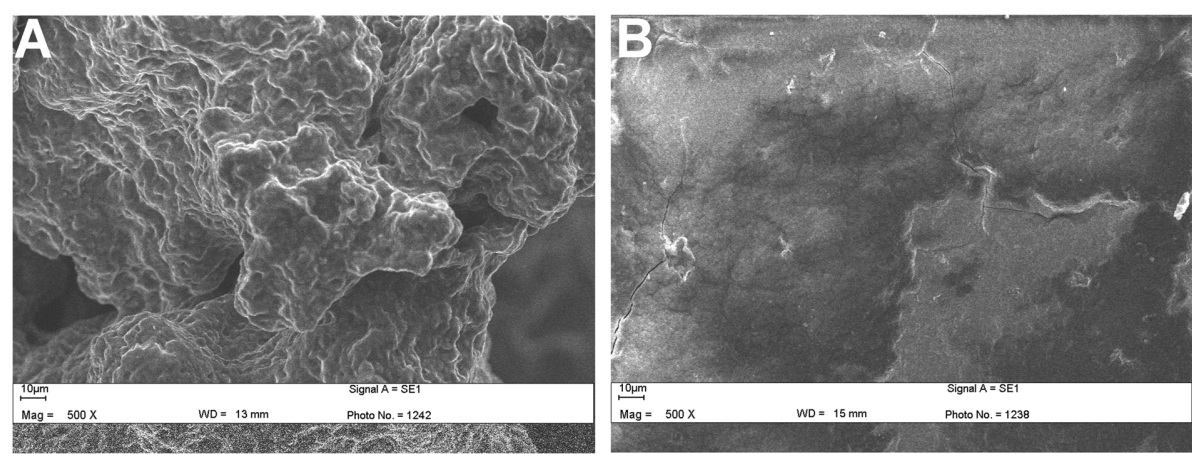

Fig. 5. Scanning electron micrographs. (A) Thai water kefir grain. (B) Crude EPS from A. pasteurianus WS3. Magnification at 500X.

\section{Discussion}

This is the first report on finding yeast $M$. guilliermondii in microbial strains of kefir. M. guilliermondii has been shown to have antifungal properties, producing aromatic flavors during the bread-making process [26] and is found in fermented drinks made by malt, corn and oats called 'Boza' in Eastern Europe [27] indicating it may play a role in producing unique aromas and also alcohol in kefir drinks. In addition, our finding concurred with previous reports that showed LAB including L. casei, L. higardii, L. hordei, L. nagelii, L. citreum, $L$. mesenteroides [28], L. lactis subsp. cremoris H414 [29], and L. lactis subsp. cremoris B891 [30] as EPS producers. In addition, $A$. fabarum and $A$. syzygii were found in German kefir and A. orientalis in Brazilian milk kefir [4]; however, in this work A. tropicalis was identified indicating its presence in kefir of a tropical country. $A$. tropicalis was also found to produce EPS [31]. In the previous report, Tibetan milk kefir that contained 15 culturable microbes including yeasts (Kazachstania unispora and S. cerevisiae), AAB (A. okinawensis) and LAB (Leu. pseudomesenteroides and L. lactis subsp. lactis) [23]. Only certain strains of $A$. okinawensis, $K$. unispora and 
Leu. pseudomesenteroides from all reported strains were able to produce EPS. This indicated that this EPS-producing capacity seems to differ at the subspecies level. In addition, L. lactis subsp. lactis did not produce EPS from skim milk containing lactose as a carbon source [23]. Similarly, our L. lactis WS17 only produced EPS upon sucrose and glucose, but not lactose (Fig. 3). Similar to the previous study [14], sucrose seemed to be the most efficient sugar substrate, producing EPS with the largest diameter on the agar plates by most bacteria, while lactose was the least efficient, producing the smallest sizes of EPS. These EPSs were thought to be important in the formation and integrity of kefir grains. The type of sugar substrate has a huge impact on EPS productivity and may also influence the composition of EPS and hence its antioxidant activity and anti-tyrosinase activity. Strain characteristics may also be attributed to EPS production. Previously it was shown that EPS production by LAB depended on the sugar substrate present in the culture medium and its regulation of the EPS biosynthetic pathway [32, 33]. Inhibition of the activity of some key enzymes for EPS synthesis caused by certain sugar derived metabolites might also decrease EPS synthesis [34]. This may be why lactose was the least efficient sugar for EPS production in our case.

Molecules responsible for antioxidant activity in the bacterial cell-free supernatants could be extracellular EPSs. Monosaccharides including galactose, glucose, rhamnose and mannose are effective reductive agents as they have a hidden aldehyde moiety [35]. The radical scavenging activity of our EPS, via electron transfer and hydrogen atom transfer mechanisms from FRAP and $\mathrm{ABTS}^{\cdot+}$ assays, respectively may be based on their reductive nature. This might be attributed to the hydroxyl group and other functional groups in EPS, such as $-\mathrm{COOH}, \mathrm{C}=\mathrm{O}$ and -O-, which can donate electrons to reduce the radicals to a more stable form, or react with the free radicals to terminate the radical chain reaction [36].

The EPS production yield by Acetobacter sp. WS3 and L. casei WS13 was higher than those reported recently by other researchers [22, 31, 38-44]. EPS production by Acetobacter sp. SKU1100 isolated from fruits in Thailand, under static culture condition reached a maximum of $1,230 \mathrm{mg} \mathrm{EPS} / \mathrm{L}$ at $37^{\circ} \mathrm{C}$ after 3 days of cultivation using $0.5 \%$ glucose and $10 \%$ potato extract as substrates
[31]. A. okinawensis RJ-4 from Tibetan kefir produced approximately $380 \mathrm{mg}$ EPS/L from lactose within $24 \mathrm{~h}$ [22]. L. casei LC2W with probiotic effects showed EPS production at $150.3 \mathrm{mg} / \mathrm{l}$ from 32 -h fermentation in MRS medium with $2 \%$ glucose [38]. A considerable variation in EPS production was observed in $L$. casei depending on sugar substrate used and culture conditions. For example, L. casei CG11 produced EPS at 130-250 mg/l with lactose as the poorest carbon source and glucose as the most efficient in basal minimum medium [39], while Mozzi et al. reported EPS production of $488 \mathrm{mg} / \mathrm{l}$ for $L$. casei [40]. Bioactivities of the EPS in this study were similar to the previous findings indicating that the partially purified EPS from L. sakei Probio 65 (10-200 mg/ $\mathrm{ml}$ ) exhibited potent efficacy on inhibiting tyrosinase enzyme by $13.17-62.85 \%$ and displayed a considerable amount of antioxidant efficacy on scavenging DPPH by 49.56\% [41]. The hydroxyl groups in EPS structure could form a hydrogen bond to the active site of tyrosinase enzyme, resulting in steric hindrance or changed conformation and thus causing it to have a lower catalytic activity [42]. Our A. pasteurianus WS3 EPS showed anti-tyrosinase activity of $20.35 \%$ which was much higher than that of EPS from the mushroom Fomitopsis feei $(2.13,4.89,8.84 \%$ tyrosinase inhibition from 25,50 and $100 \mathrm{mg} / \mathrm{ml}$ extract) [43]. Similar to our finding, EPSs (1-10 mg/ml) from Bacillus licheniformis strains isolated from Kimchi exhibited anti-tyrosinase activities in a range of $22.5-37.0 \%$ which was significantly higher than that of beta-glucan (6.0-17.0\%) at the same concentrations [44].

The reducing potentials of EPS antioxidants are linked with their electron donating abilities of semi-acetal at $\mathrm{C} 1$ of the reducing end of a polysaccharide and thus can reduce $\mathrm{Fe}^{3+}$ to $\mathrm{Fe}^{2+}$. In comparison with the previous report [45], three EPSs from endophytic bacterium Paenibacillus polymyxa EJS-3 exhibited FRAP values of $10.79,3.37$, and $4.38 \mathrm{mM} \mathrm{FeSO}_{4} / \mathrm{g}$ which were over 10-fold lower those found in our EPSs ranging from 132-280 mM $\mathrm{FeSO}_{4} / \mathrm{g}$ (Table 3) suggesting stronger reducing power. However, the positive control, ascorbic acid, showed a much higher FRAP value of $11,919 \mathrm{mM}$ $\mathrm{FeSO}_{4} / \mathrm{g}$, suggesting that its antioxidant power was much stronger than that of EPSs in their work and our work. The appearance and structure of our A. pasteurianus WS3 EPS by SEM analysis is similar to the EPS 
produced by previously reported strain A. okinawensis RJ-4 [22] exhibited a compact structure with the appearance of a smooth surface with a thin film covering, indicating specific characteristics of materials used to make plasticized films [46]. Other appearances of bacterial EPSs can also be observed. For example, B. tequilensis PS21 had a grainy appearance with an uneven surface, covered with pores [47].

To conclude, our findings have shed light on microbial strains, EPS producers of Thai water kefir as distinct from Thai milk kefir and have shown differences in microbial strains with kefirs from other countries. These differences make the kefir products of each country very different in terms of flavor and health benefits. Microbes isolated from our study might provide a fundamental information for developing new kefir grains with desired properties as novel functional foods. Their EPS-producing capacity also offers antioxidant activity and antityrosinase activity for future potential biotechnological applications including food additives, animal feed, cosmetic ingredients, and biodegradable films.

\section{Acknowledgments}

This research was financially supported by Mahasarakham University, Thailand (Fast track 2019 research fund) with grant no. 5901011. The authors would like to thank Ploynamthip Puykamsing, Pichaya Thangtong, Thunyaporn Chompoowiset, Supawat Tipparak, Kwanthida Chachai, Kanokwan Wanchoosert and Puntiwa Srisupan for their help and assistance in this project and also Peter Humphrey Charge for proofreading the manuscript.

\section{Conflict of Interest}

The authors have no financial conflicts of interest to declare.

\section{References}

1. Nielsen B, Gürakan GC, Ünlü G. 2014. Kefir: a multifaceted fermented dairy product. Probiotics Antimicrob. Proteins. 6: 123-135.

2. John SM, Deeseenthum S. 2015. Properties and benefits of kefir A review. Songklanakarin J. Sci. Technol. 37: 275-282.

3. Diosma G, Romanin DE, Rey-Burusco MF, Alejandra L, Garrote LG. 2014. Yeasts from kefir grains: isolation, identification, and probiotic characterization. World J. Microbiol. Biotechnol. 30: 43-53.

4. Miguel MG, Cardoso PG, Magalhaes-Guedes KT, Schwan RF. 2013. Identification and assessment of kefir potential for sugar/ ethanol-resistance. Braz. J. Microbiol. 44: 113-118.

5. Silva KR, Rodrigues SA, Filho LX, Lima AS. 2009. Antimicrobial activity of broth fermented with kefir grains. Appl. Microbiol. Biotechnol. 152: 316-325.

6. Waldherr FW, Doll VM, Meißner D, Vogel RF. 2010. Identification and characterization of a glucan-producing enzyme from Lactobacillus hilgardii TMW 1.828 involved in granule formation of water kefir. Food Microbiol. 27: 672-678.

7. Mitsue T, Tachibana K, Fujio Y. 1999. Efficient kefiran production by a mixed culture of Lactobacillus kefiranofaciens KF-75 and yeast strains. J. Biosci. Bioeng 87: 400.

8. Deeraksa A, Moonmangmee S, Toyama H, Yamada M, Adachi O, Matsushita K. 2005. Characterization and spontaneous mutation of a novel gene, polE, involved in pellicle formation in Acetobacter tropicalis SKU1100. Microbiology 151: 4111-4120.

9. Freitas F, Alves AV, Carvalheira M, Costa N, Oliveira R, Reis MA. 2009. Emulsifying behaviour and rheological properties of the extracellular polysaccharide produced by Pseudomonas oleovorans grown on glycerol by product. Carbohydr. Polym. 78: 549556.

10. Mahapatra S, Banerjee D. 2013. Fungal Exopolysaccharide: production, composition and applications. Microbiol. Insights. 6: 116.

11. Parikh A, Madamwar D. 2006. Partial characterization of extracelIular polysaccharides from Cyanobacteria. Bioresour. Technol. 97: 1822-1827.

12. Sutherland IW. 2001. Microbial polysaccharides from gram-negative bacteria. Int. Dairy J. 11: 663-674.

13. Mota R, Guimaraes R, Buttel Z, Rossi F, Colica G, Silva CJ, et al. 2013. Production and characterization of extracellular carbohydrate polymer from Cyanothece sp. CCY 0110. Carbohydr. Polym. 92: 1408-1415.

14. Luang-In V, Deeseenthum S. 2016. Exopolysaccharide-producing isolates from Thai milk kefir and their antioxidant activities. LWTFood Sci. Technol. 73: 592-601.

15. Kimura M. 1980. A simple method for estimating evolutionary rate of base substitutions through comparative studies of nucleotide sequences. J. Mol. Evol. 16: 111-120.

16. Kumar S, Stecher G, Tamura K. 2016. MEGA7: Molecular evolutionary genetics analysis version 7.0 for bigger datasets. Mol. Biol. Evol. 33: 1870-1874.

17. Rambaut A. 2012. FigTree v1. 4. Molecular evolution, phylogenetics and epidemiology. Edinburgh, UK: University of Edinburgh, Institute of Evolutionary Biology. Biology. <http://tree.bio.ed. ac.uk/software/figtree/>.

18. Paulo EM, Vasconcelos MP, Oliveira IS, Affe HMJ, Nascimento R, MELO, I. S. de et al. 2012. An alternative method for screening lactic acid bacteria for the production of exopolysaccharides with rapid confirmation. Ciência e Tecnologia de Alimentos. 32: 710714.

19. Benzie IFF, Strain JJ. 1996. The ferric reducing ability of plasma (FRAP) as a measure of antioxidant power : The FRAP Assay. Anal. Biochem. 239: 70-76.

20. Seeram NP, Adams LS, Zhang Y, Lee R, Sand D, Scheuller HS, et al. 2006. Blackberry, black raspberry, blueberry, cranberry, red rasp- 
berry, and strawberry extracts inhibit growth and stimulate apoptosis of human cancer cells in vitro. J. Agric. Food Chem. 54: 9329-9339.

21. Choi HK, Lim YS, Kim YS, Park SY, Lee CH, et al. 2008. Free-radicalscavenging and tyrosinase-inhibition activities of Cheonggukjang samples fermented for various times. Food Chem. 106: 564568.

22. Li L, Wieme A, Spitaels F, Balzarini T, Nunes O, Manaia CM, et al. 2014. Acetobacter sicerae sp. nov., isolated from cider and kefir, and identification of strains of the genus Acetobacter by dnaK, groEL and rpoB sequence analysis. Int. J. Syst. Evol. Microbiol. 64: 2407-2415.

23. Chen Z, Shi J, Yang X, Liu Y, Nan B, Zhongfu W. 2016. Isolation of exopolysaccharide-producing bacteria and yeasts from Tibetan kefir and characterisation of the exopolysaccharides. Int. J. Dairy Technol. 69: 410-417.

24. Wang Y, Xu N, Xi A, Ahmed Z, Zhang B, Bai X. 2009. Effects of Lactobacillus plantarum MA2 isolated from Tibet kefir on lipid metabolism and intestinal microflora of rats fed on high-cholesterol diet. Appl. Microbiol. Biotech. 84: 341-347.

25. Rao BP, Sudharsan K, Sekaran RCHG, Mandal AB. 2013. Characterization of exopolysaccharide from Bacillus amyloliquefaciens BPRGS for its bioflocculant activity. Int. J. Sci. Eng. Res. 4: 16961704.

26. Coda R, Rizzello CG, Di Cagno R, Trani A, Cardinali G, Gobbetti M. 2013. Antifungal activity of Meyerozyma guilliermondii: identification of activity compounds synthesized during dough fermentation and their effect on long-term storage of wheat bread. Food Microbiol. 33: 243-251.

27. Osimani A, Garofalo C, Aquilanti L, Milanovic V, Clementi F. 2015. Unpasteurised commercial boza as a source of microbial diversity. Int. J. Food Microbiol. 194: 62-70.

28. Gulitz A, Stadie J, Wenning M, Ehrmann MA, vogel RF. 2011. The microbial diversity of water kefir. Int. J. Food Microbiol. 151: 284288.

29. Gruter M, Leeflang BR, Kuiper J, Kamerling JP, Vliegenthart JFG. 1992. Structure of the exopolysaccharide produced by Lactococcus lactis sub cremoris H414 grown in a defined medium or skimmed-milk. Carbohydr. Res. 231: 273-291.

30. van Casteren WHM, de Waard P, Dijkema C, Schols HA, Voragen AGJ. 2000. Structural characterisation and enzymatic modification of the exopolysaccharide produced by Lactococcus lactis subsp. cremoris B891. Carbohydr. Res. 327: 411-422.

31. Ali IAl, Akakabe $Y$, Moonmangmee S, Deeraksa A, Matsutani M, Yakushi T. 2011. Structural characterization of pellicle polysaccharides of Acetobacter tropicalis SKU1100 wild type and mutant strains. Carbohydr. Polym. 86: 1000-1006.

32. Grobben GJ, Smith MR, Sikkema J, de Bont JAM. 1996. Influence of fructose and glucose on the production of exopolysaccharides and the activities of enzymes involved in the sugar metabolism and the synthesis of sugar nucleotides in Lactobacillus delbrueckii subsp. Bulgaricus NCFB 2772. Appl. Microbiol. Biotechnol. 46: 279-284.
33. Looijesteijn PJ, Boels IC, Kleerebezem M, Hugenholtz J. 1999. Regulation of exopolysaccharide production by Lactococcus lactis subsp. cremoris by the sugar source. Appl. Environ. Microbiol. 65: 5003-5008.

34. Zhang T, Zhang C, Li S, Zhang Y, Yang Z. 2011. Growth and exopolysaccharide production by Streptococcus thermophilus ST1 in skim milk. Braz. J. Microbiol. 42: 1470-1478.

35. Yang JH, Mau JL, Ko PT, Huang LC. 2000. Antioxidant properties of fermented soybean broth. Food Chem. 71: 249-54.

36. Sakanaka S, Tachibana Y, Okada Y. 2005. Preparation and antioxidant properties of extracts of Japanese persimmon leaf tea (kakinoha-cha). Food Chem. 89: 569-675.

37. Moonmangmee S, Toyama H, Adachi O, Theeragool G, Lotong N, Matsushit K. 2002. Purification and characterization of a novel polysaccharide involved in the pellicle produced by a thermotolerant Acetobacter strain. Biosci. Biotechnol. Biochem. 66: 777-783.

38. Li N, Wang Y, Zhu P, Liu Z, Guo B, Ren J. 2015. Improvement of exopolysaccharide production in Lactobacillus casei LC2W by overexpression of NADH oxidase gene. Microbiol. Res. 171: 73-77.

39. Cerning J, Renard CM, Thibault JF, Bouillanne C, Landon M, Desmazeaud $M$ et al. 1994. Carbon source requirements for exopolysaccharide production by Lactobacillus casei CG11 and partial structure analysis of the polymer. Appl. Environ. Microbiol. 60: 3914-3919.

40. Mozzi F, Savoy de Giori G, Oliver G, Font de Valdez G. 1996. Exopolysaccharide production by Lactobacillus casei in milk under different growth conditions. Milchwissenschaft. 51: 670-673.

41. Bajpai VK, Rather IA, Park YH. 2016. Partially purified exo-polysaccharide from Lactobacillus sakei Probio 65 with antioxidant, aglucosidase and tyrosinase inhibitory potential. J. Food Biochem. 40: $264-274$.

42. Baek HS, Rho HS, Yoo JW, Ahn SM, Lee JY, Kim JA. 2008. The inhibitory effect of new hydroxamic acid derivatives on melanogenesis. Bull. Korean Chem. Soc. 29: 43-46.

43. Hima Bindu NSVSSSL, Singara Charya MA. 2017. Biological activities of exopolysaccharide from the mushroom. Fomitopsis feei. Int. J. Microbiol. Res. 8: 48-58.

44. Song YR, Song NE, Kim JH, Nho YC, Baik SH. 2011. Exopolysaccharide produced by Bacillus licheniformis strains isolated from Kimchi. J. Gen. Appl. Microbiol. 57: 169-175.

45. Liu J, Luo J, Ye H, Sun Y, Lu Z, Zeng X. 2010. In vitro and in vivo antioxidant activity of exopolysaccharides from endophytic bacterium Paenibacillus polymyxa EJS-3. Carbohydr. Polym. 82: 12781283.

46. Ahmed Z, Wang Y, Anjum N, Ahmad A, Khan ST. 2013. Characterization of exopolysaccharide produced by Lactobacillus kefiranofaciens ZW3 isolated from Tibet kefir-Part II. Food Hydrocoll. 30: 343-350.

47. Luang-In V, Saengha W, Deeseenthum, S. 2018. Characterization and bioactivities of a novel exopolysaccharide produced from lactose by Bacillus tequilensis PS21 isolated from Thai milk kefir. Microbiol. Biotechnol. Lett. 46: 9-17. 